\title{
A Balloon Filled with Nitrogen Gas Does Not Satisfy the Air- or Moisture-Free Reaction Condition
}

\author{
Kwang-Jin Hwang, Seung Hee Lee, Hyung Jin Kim, ${ }^{\dagger}$ Jee-Yon Lee, ${ }^{\S}$ and Jin Seog Kim*,** \\ Department of Chemical System Engineering, Hongik University at Jochiwon, Chungnam 339-701, Korea \\ ${ }^{\dagger}$ Center for Functional Nano Fine Chemicals, School of Applied Chemical Engineering, \\ Chonnam National University, Gwangju 500-757, Korea \\ ${ }^{\ddagger}$ Division of Metrology for Quality of Life, Korea Research Institute of Standards and Science, Deajeon 305-600, Korea \\ ${ }^{*}$ E-mail: jkim@kriss.re.kr \\ ${ }^{\S}$ Emergency Preparedness \& Environment Devision, Korea Institute of Nuclear Safety, Daejeon 305-338, Korea \\ Received January 7, 2010, Accepted January 29, 2010
}

Key Words: Balloon, Anhydrous, Moisture-free condition, Organometallics

In the basic reaction media using Grignard reagents, alkyllithum, metal hydrides and organometallic reagents, water can act as a strong acid to ruin the reagents. ${ }^{1}$ On the other hand, in the reaction using Lewis acids such as thionyl halide, phosphorous halide, boron halides, or organoaluminium compounds, water can act as a nucleophile that damages those reagents. Due to these harmful properties of water, synthetic researchers have focused extensive attention on technical methods to retain an anhydrous or air-free condition. Although the Schlenk technique $^{2}$ and glove box ${ }^{3,4}$ can be used to accomplish such conditions precisely and perfectly, tedious installation and careful utilization processes are required. Therefore, the convenient and easier method of using a rubber balloon filled with nitrogen gas has been considered and used to maintain an anhydrous condition for air-sensitive reactions. ${ }^{5,6}$

In general, a balloon filled with inert gas connected to the reaction flask excludes the outer atmosphere from the reactor due to the higher inside pressure than that outside the reactor. Helium gas in a balloon is, however, reported to be exchanged with the external humidity due to gas permeation. ${ }^{7}$ We wondered whether a reactor could be protected only by a balloon filled with inert gas to the extent of satisfying the anhydrous condition. To confirm the possibility, we analyzed the gases in a nitrogen gas-filled balloon in a laboratory. The moisture content in the reactor connected with the nitrogen gas-filled balloon was analyzed by an IR spectrophotometer. The aim of this study was to alert researchers using a nitrogen gas-filled balloon to satisfy the anhydrous reaction condition, of the danger of outside moisture permeating very rapidly into the nitrogen gas balloon.

A schematic of the experimental setup is shown in Figure 1. The gas in the balloon was analyzed by an IR spectrometer for 60 minutes, as shown in Figure 2. In the IR analysis of the air, the absorption bands of $\mathrm{H}_{2} \mathrm{O}$ appeared in the ranges of 3560 3928 and $1351 \sim 1905 \mathrm{~cm}^{-1}$ and that of $\mathrm{CO}_{2}$ in the range of $2371 \sim 2307 \mathrm{~cm}^{-1}$. The nitrogen gas (purity $>99.999 \%$ ) used to fill the balloon did not show any trace of $\mathrm{H}_{2} \mathrm{O}$ or $\mathrm{CO}_{2}$, in complete contrast to the air in the IR analysis, as shown in Figure 2 (a). As depicted in Figure 2 (b-f), the absorption intensities of both $\mathrm{H}_{2} \mathrm{O}$ and $\mathrm{CO}_{2}$ increased with time. To determine the relative increases of $\mathrm{H}_{2} \mathrm{O}$ and $\mathrm{CO}_{2}$ contents in the

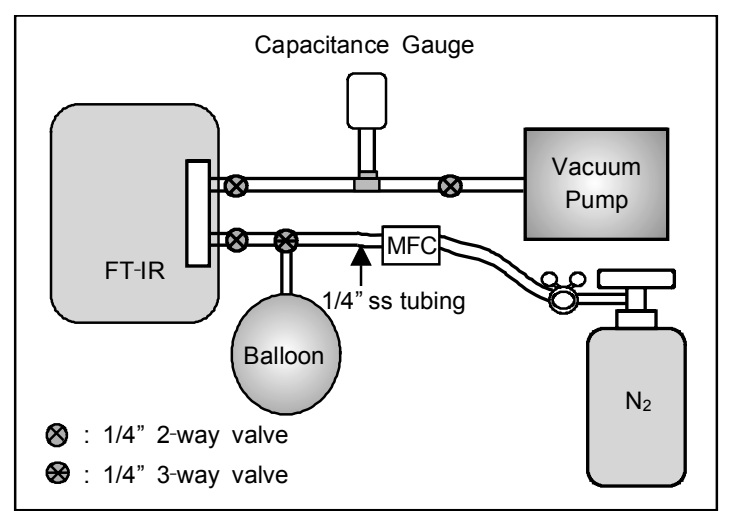

Figure 1. Schematic diagram of the apparatus for analyzing the gas content in the nitrogen gas-filled balloon using an FT-IR spectrometer (MFC: Mass flow controller).

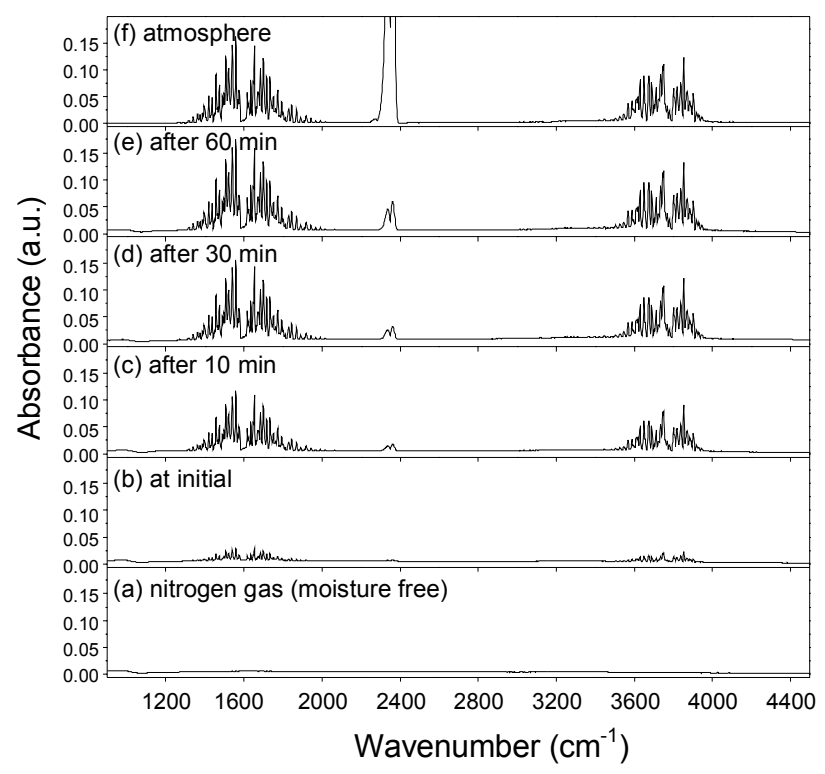

Figure 2. FT-IR spectra of the $\mathrm{N}_{2}$ gas used to fill the balloon (a), the gas inside the balloon at the time after inflation (b), and after $10 \mathrm{~min}$ (c), $30 \mathrm{~min}$ (d), and $60 \mathrm{~min}$ (e). The absorption bands for $\mathrm{H}_{2} \mathrm{O}$ appeared in the range of $3560-3928$ and $1351-1905 \mathrm{~cm}^{-1}$, and those for $\mathrm{CO}_{2}$ in the range of $2307-2371 \mathrm{~cm}^{-1}$. 


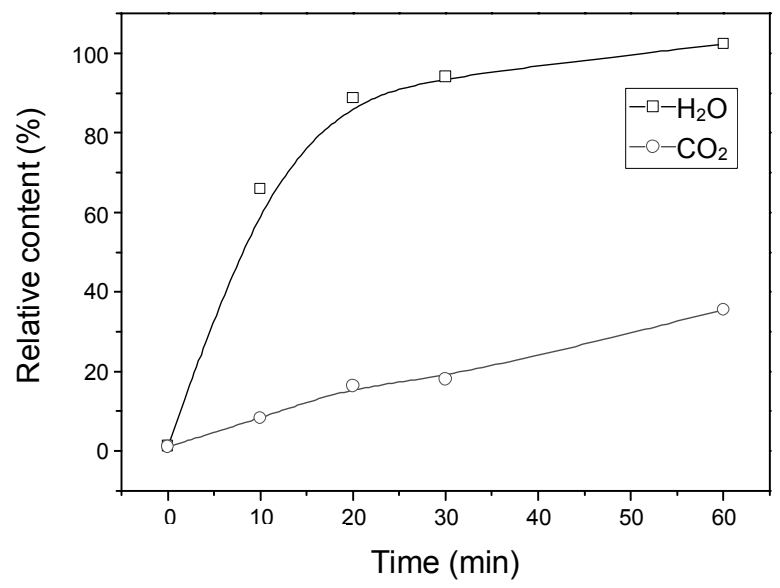

Figure 3. Relative $\mathrm{H}_{2} \mathrm{O}$ and $\mathrm{CO}_{2}$ contents in the balloon according to time. The relative gas contents were calculated based on the integration of IR absorption peaks at $1351-1905 \mathrm{~cm}^{-1}$ for $\mathrm{H}_{2} \mathrm{O}$ and $2307-2371$ $\mathrm{cm}^{-1}$ for $\mathrm{CO}_{2}$.

Table 1. Calculations of FT-IR peak areas of $\mathrm{H}_{2} \mathrm{O}$ and $\mathrm{CO}_{2}$ in the $\mathrm{N}_{2}$ gas used to fill the ballon, in the balloon at various time intervals, and in the atmosphere.

\begin{tabular}{ccccc}
\hline \multirow{2}{*}{ Gas } & Time (min) & \multicolumn{3}{c}{ Band region $\left(\mathrm{cm}^{-1}\right)$} \\
\cline { 3 - 5 } & & $\begin{array}{c}3560-3928 \\
\left(\mathrm{H}_{2} \mathrm{O}\right)\end{array}$ & $\begin{array}{c}1351-1905 \\
\left(\mathrm{H}_{2} \mathrm{O}\right)\end{array}$ & $\begin{array}{c}2307-2371 \\
\left(\mathrm{CO}_{2}\right)\end{array}$ \\
\hline $\mathrm{N}_{2}$ & - & 0.054 & 0.0022 & 0.00078 \\
In balloon & 0 & 1.29 & 2.52 & 0.0197 \\
In balloon & 10 & 6.38 & 11.63 & 0.154 \\
In balloon & 20 & 8.59 & 15.68 & 0.306 \\
In balloon & 30 & 9.11 & 16.62 & 0.386 \\
In balloon & 60 & 9.88 & 18.07 & 0.662 \\
Atmosphere & - & 9.75 & 17.65 & 1.864 \\
\hline
\end{tabular}

balloon, the moisture quantities in the balloon are shown in Figure 3 based on the integration of the absorption peaks in the region of $1351 \sim 1905 \mathrm{~cm}^{-1}$ corresponding to the symmetric bending normal mode of $\mathrm{H}_{2} \mathrm{O}$ and compared with the atmospheric moisture content. The $\mathrm{CO}_{2}$ content in the balloon was also obtained from the integration of the absorption peaks in the range of $2307 \sim 2371 \mathrm{~cm}^{-1}$. As shown in Figure 3, the water content in the balloon increased rapidly to $14 \%$ of atmospheric water concentration even immediately after inflating the balloon with nitrogen. The water concentration in the balloon reached $65 \%$ of atmospheric water concentration in 10 minutes and almost reached equilibrium with the atmosphere after 30 minutes.

However, $\mathrm{CO}_{2}$ gas permeated into the balloon more slowly than water, which was attributed to its affinity to the rubber surface and heavier molecular weight according to Graham's law. ${ }^{8}$ The theoretical permeation rate of $\mathrm{CO}_{2}$ was estimated as about 1.5 times slower than that of $\mathrm{H}_{2} \mathrm{O}$. Grignard reagents can react with $\mathrm{CO}_{2}$ to be converted into the corresponding carboxylic acids. For prolonged Grignard reactions, therefore, diffu- sion of $\mathrm{CO}_{2}$ into a balloon was quite noticeable and could affect the reaction because the $\mathrm{CO}_{2}$ content was increased to $35 \%$ of the atmospheric $\mathrm{CO}_{2}$ after 60 minutes.

In summary, analysis of the gas in a nitrogen gas-filled balloon indicated that the penetration of water molecules into the balloon achieved a moisture content of $65 \%$ of atmospheric water concentration in 10 minutes. The results presented here should alert synthetic chemists in organic or inorganic laboratories to the inability of an inert gas-filled balloon to provide a moisture-free or air-free condition in the reaction system.

\section{Experimental Section}

Thick-wall rubber balloons were purchased from Aldrich Chemical Co. The balloon was tied by a rubber band at a part of a three-way valve leading to the inlet port of an FT-IR spectrometer. The balloon was inflated and deflated five times with high purity $\mathrm{N}_{2}$ (99.999\%, Deokyang Energen Co., Korea) to remove the moisture physically adsorbed inside the balloon. The moisture content remaining in the balloon filled with a constant pressure of nitrogen ( $c a .1 .5$ liter) was analyzed by a BRUKER IFS 120 HR FT-IR spectrometer after inflation at atmospheric pressure. A sample holder of a stainless steel IR cell equipped with a $\mathrm{KBr}$ window was used. The data were recorded by accumulating 30 scans with a resolution of $4 \mathrm{~cm}^{-1}$ under a constant temperature of $20 \pm 1{ }^{\circ} \mathrm{C}$. A capacitance gauge attached to the vacuum line was used to confirm the inner pressure and to check for leakage in the IR cell. Data acquisition and qualitative and quantitative analyses of the IR spectra were performed using an analysis program (OPUS NT spectroscopic software) and the peak areas of $\mathrm{H}_{2} \mathrm{O}$ and $\mathrm{CO}_{2}$ in the balloon were calculated as shown in Table 1.

Acknowledgments. This work was funded by the Korea Metrological Administration Research and Development Program.

\section{References}

1. For the chemical properties of reagents see: Encyclopedia of Reagents for Organic Synthesis; Paquette, L. A., Ed.; John Wiley: New York, 1995.

2. Errington, R. J. Advanced Practical Inorganic and Metalorganic Chemistry; Chapman \& Hall: London, 1997, pp 33-39.

3. Schriver, D. F.; Drezdzon, M. A. The Manipulation of Air-Sensitive Compounds; 2nd ed.; Wiley: New York, 1986.

4. Errington, R. J. Advanced Practical Inorganic and Metalorganic Chemistry; Chapman \& Hall: London, 1997, pp 53-66.

5. Mayo, D. W.; R. M. Pike; Trumper, P. K. Microscale Organic Laboratory with Multistep and Multiscale Synthesis; 3rd ed.; Wiley \& Sons, Inc.: 1994; Chap. 3, pp 30-31.

6. Leonard, J.; Lygo, B.; Procter, G. Advanced Practical Organic Chemistry; 2nd ed.; Blackie Academic \& Professional: Glasgow, 1995; pp 140-143.

7. Lee, J.-Y.; Yoo, H.-S.; Park, J. S.; Hwang, K.-J.; Kim, J. S. J. Chem. Edu. 2005, 82, 288.

8. Deese, W. C.; Washburn, A. M. J. Chem. Edu. 1996, 73, 539. 